Boletín de la Sociedad Botánica de México 48: 119-134, 1988

DOI: $10.17129 /$ botsci.1350

Bol. Soc. Bot. México 48: 119-134 (1988)

\title{
El maguey (Agave, subgénero Agave) en el altiplano potosino-zacatecano ${ }^{1}$
}

\author{
J. Jesús Tello Balderas ${ }^{2}$ y Edmundo García Moya ${ }^{3}$
}

\begin{abstract}
RESUMEN. Esta investigación concierne a la identidad taxonómica, distribución geográfica y ecológica regionales, las formas de utilización y el conocimiento empírico de los agaves del subgénero Agave en San Luis Potosí y Zacatecas. Se encontraron las siguientes especies y subespecies silvestres: Agave salmiana ssp. crassispina y $A$. scabra ssp. potosiensis; $A$. parrasana; semicultivadas: $A$. americana var. oaxacensis; cultivadas: $A$. mapisaga var. mapisaga y $A$. salmiana var. salmiana (tres formas). A. salmiana ssp. crassispina es la más ampliamente distribuida y la más abundante. Se desarrolla entre 1800 y $2400 \mathrm{msnm}$; en sustratos coluvio-aluvial (ígneo y sedimentario); suelo litosol eútrico. Es elemento importante del matorral crasicaule y matorral micrófilo. Es la única especie utilizada en la elaboración de mezcal y quiote, y es la utilizada en mayor cantidad como forraje. Tiene otros usos. A. mapisaga var. mapisaga y $A$. salmiana var. salmiana, se cultivan principalmente al NNW de la ciudad de San Luis Potosí, a 1880 msnm; sustrato aluvial; xerosol háplico; matorral crasicaule deteriorado; son utilizados principalmente en la extracción de aguamiel para pulque, forraje, elaboración de miel de aguamiel, construcción, ornamental y cercas vivas. Existe conocimiento empírico para un manejo favorable del recurso, pero debido a diversas situaciones, su aplicación actual es reducida.
\end{abstract}

ABSTRACT. This research deals with the taxonomy, geographical and ecological distribution, and the forms of utilization and available empirical knowledge of Agave spp., subgenus Agave, in San Luis Potosí and Zacatecas. The following taxa were found: wild species: Agave salmiana ssp. crassispina, A. scabra ssp. potosiensis, A. parrasana; semicultivated species: $A$. americana var. oaxacensis; cultivated species: $A$. mapisaga var. mapisaga, three forms of $A$. salmiana var. salmiana. A. salmiana ssp. crassispina is the most widely distributed and abundant. It is found from 1800 to $2400 \mathrm{~m}$ altitude, on igneous and sedimentary substrates, euthric Litosol, and it is an element of the cactus and desert scrubland. It is used to produce mezcal and for the preparation of the edible flowering stem (quiote). It is the Agave most used as forage, and it is also utilized as construction material, as fuel, in soil conservation, as ornament and as hedge plant. A. mapisaga var. mapisaga and $A$. salmiana var. salmiana are cultivated principally to the NNW of the

${ }^{1}$ Contribución conjunta del Centro Regional para Estudios de Zonas Áridas y Semiáridas (CREZAS-CP) y del Centro de Botánica, Colegio de Postgraduados, Chapingo, Estado de México.

${ }^{2}$ Centro de Botánica adscrito al CREZAS, Iturbide 73. Salinas de Hidalgo 78600, San Luis Potosí.

${ }^{3}$ Centro de Botánica, Colegio de Postgraduados, Chapingo 56230, Estado de México. potosino-zacatecano. Boletín de la Sociedad Botánica de México 48: 119-134. 
city of San Luis Potosí, at $1880 \mathrm{~m}$, on a deteriorated cactus scrubland. Aguamiel, pulque and honey are elaborated from the natural or fermented juice extracted from the meristem of the plant. There is considerable amount of empirical knowledge regarding the management of this resource. However due to several problems its current use is reduced.

En el altiplano potosino-zacatecano, la actividad de recolección de productos espontáneos vegetales se realiza durante todo el año. Los productos obtenidos son aprovechados en forma directa, transformados mediante tecnología casera o en pequeñas agroindustrias de la región. Tal es el caso del maguey, planta utilizada en variadas formas. Su manejo actual es deficiente, poniendo en peligro su conservación. Poco consigna la literatura sobre los principios y prácticas que fundamenten el manejo apropiado para el uso sostenido del recurso. Los objetivos del trabajo son: a) precisar su identidad taxonómica; b) conocer su distribución regional, tanto geográfica como ecológica, y c) registrar sus formas de utilización y el conocimiento empírico existente.

Descripción del área de estudio. Es parte del desierto chihuahuense y está constituida por las altiplanicies del centro y sur de San Luis Potosí y sureste de Zacatecas (Mi-

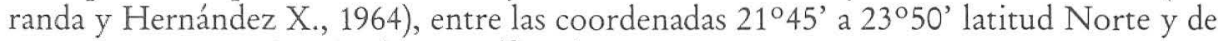
$100^{\circ} 30^{\prime}$ a $101^{\circ} 45^{\prime}$ longitud Oeste (fig. 1).

Las rocas sedimentarias afloran con mayor frecuencia, destacando por su extensión las calizas, que constituyen la mayor parte de las elevaciones montañosas. Los aluviones son de diferentes tipos, en las depresiones y llanuras áridas alcanzan enormes extensiones y espesores. Las rocas ígneas efusivas, entre ellas las riolitas, predominan en numerosas elevaciones.

El clima se caracteriza por la escasez e irregularidad de la lluvia, la coincidencia de la época lluviosa con la temporada caliente del año, la oscilación acentuada de la temperatura diurna y la evaporación muy intensa, debido a la fuerte luminosidad, la baja presión y la humedad atmosférica (Rzedowski, 1957, 1965 y 1968).

Los suelos son de origen aluvial y coluvial, de color rojizo, con $\mathrm{pH}$ ligeramente ácido (4.6 a 6.0), de textura franco-arenosa cuando son derivados de roca ígnea, o de color gris claro o café con $\mathrm{pH}$ ligeramente alcalino (7-8) y textura franco-arcillosa cuando se derivan de roca caliza (García M. y Villa V., 1977).

Rzedowski (1955, 1957 y 1965), valiéndose de criterios fisonómicos, distingue cuatro tipos principales de vegetación: a) matorral desértico micrófilo (Larrea, Flourensia y Prosopis), b) matorral desértico rosetófilo (Agave, Hechtia y Euphorbia), c) matorral crasicaule (Opuntia y Myrtillocactus), y d) zacatal (Boutelona y Mublenbergia).

\section{ANTECEDENTES}

El género Agave (Agavaceae) del griego "admirable", incluye más de 300 especies muy conspicuas en las porciones áridas y semiáridas del hemisferio norte (Bailey, 1949).

Nativo de Norteamérica, y distribuido desde Utah en el norte, hasta Centroamérica y el Caribe en el sur, México es el centro de su diversificación. El género Agave com- 


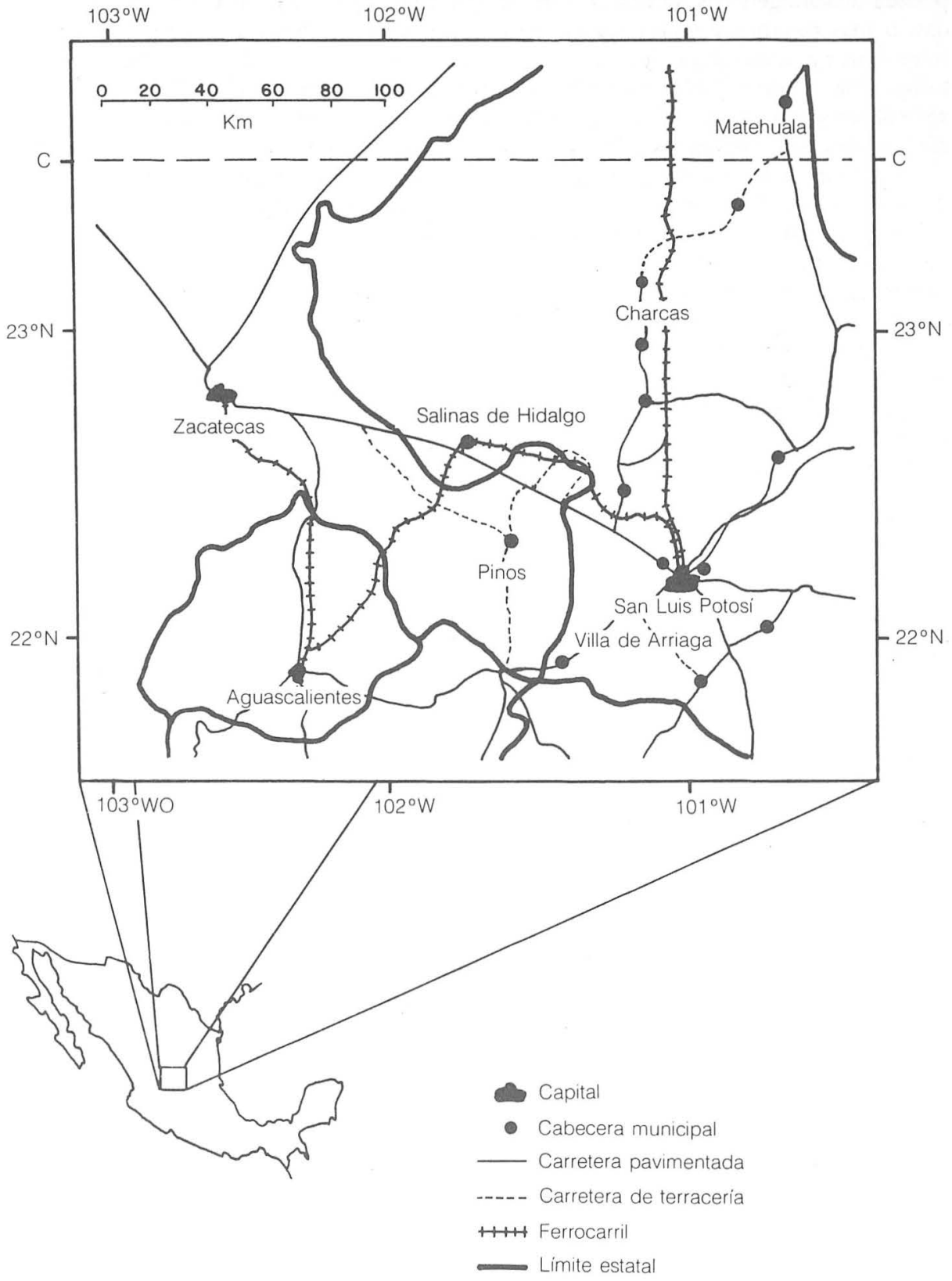

Fig. 1. Localización del área de estudio. 
prende dos subgéneros naturales: Littaea, que tiene flores espigadas en pares o agrupadas, o más raramente racimosas en pequeños grupos distintos y Agave (Euagave) que tiene flores paniculadas en grandes grupos umbelados sobre pedúnculos laterales (Breitung, 1968; Gentry, 1982). Este último autor reconoce 136 especies, 25 subespecies, 29 variedades y 7 formas, 197 taxa para el continente americano, agrupadas en 8 y 12 secciones para los subgéneros Littaea y Agave, respectivamente.

Las plantas de Agave se propagan por semilla, vástagos vegetativos y propágulos producidos en la inflorescencia. La forma de propagación depende de la especie.

Según la importancia económica de sus productos, los agaves se dividen en tres grupos: los textileros, los pulqueros y los mezcaleros. Esta clasificación encuentra correspondencia regional según el grupo utilizado como fundamento económico por largo tiempo (Lezama M., 1975; Ruvalcaba M., 1983).

El maguey y el hombre desarrollaron en Mesoamérica un verdadero mutualismo; el maguey dando al hombre bebida, alimento, vestido, herramienta, protección y material para escritura; el hombre proporcionándole un ambiente de cultivo cuidadoso y esmerado y un manejo bajo el cual fue mejorando por hibridación y selección. Ciertamente, la naturaleza nunca juntó en forma tan compacta tantos elementos para el bienestar humano y la civilización (Gentry, 1976).

El pulque y el mezcal eran consumidos intensamente por los indígenas durante la Colonia (Cue C., 1979; Guerrero G., 1980). El mezcal se desarrolló a partir de la llegada de los españoles al continente, quienes habiendo introducido el proceso de destilación lo aplicaron al fermento inicial obtenido del tronco del mezcal (Bazant, 1975). Bye (1979), al rescatar las notas de campo del colector botánico E. Palmer, da cuenta del aprovechamiento del maguey para la elaboración de mezcal y pulque, principalmente en el altiplano potosino. A la fecha, la industria mezcalera y pulquera sigue aportando grandes beneficios económicos (Guerrero G., 1980).

El futuro económico del maguey dependerá de la industrialización y la utilización integral de la planta, mediante nuevas técnicas de transformación, algunas de las cuales ya se llevan a la práctica en plantas piloto, obteniéndose mieles alimenticias e industriales, pulque a granel y embotellado, refrescos y bebidas refrescantes, destilados de $40^{\circ} \mathrm{GL}$, levadura para uso pecuario y humano, jarabes de fructosa, inulina industrial, fructosanas; biomasa proteínica para uso pecuario, humano e industrial; melaza para forraje y otros usos; fibras para costalería, jarciería y artesanías (Sánchez-Marroquín, 1979). En la actualidad, estas innovaciones están fuera del alcance de los que comúnmente explotan el maguey y por lo mismo no se adoptan como práctica usual (Ruvalcaba M., 1983).

\section{Materiales y MÉTODOS}

Recorridos exploratorios y colecta de material para herbario. Con base en la bibliografía y cartografía del Cetenal 1970-1973, 1976-1977, se hicieron recorridos dentro del área de estudio, colectando ejemplares para herbario, anotando en fichas de colecta las características ambientales, morfológicas y de uso de la planta. El material colectado se herborizó y se envió para su identificación taxonómica al Herbario-Hortorio del Centro de Botánica del Colegio de Postgraduados (CHAPA) y a la Escuela Nacional de Ciencias 
Biológicas del Instituto Politécnico Nacional (ENCB). Posteriormente los ejemplares se incorporaron al Herbario del CREZAS-CP.

Registro del conocimiento empírico de los campesinos. La entrevista dirigida al hecho concreto, con informantes seleccionados por muestreo de juicio, siguió el método general de trabajo planteado por Hernández X. y Ramos (1977), y Aguirre R., J.R., (comunicación personal), el cual se basó en las siguientes consideraciones: a) mayor objetividad de la información, pues la entrevista se apoya en un hecho presente y es posible verificar dicha información; b) mayor profundización sobre el hecho descrito: la conversación abierta y la ausencia de restricciones de la precodificación, permiten registrar al máximo los aspectos del hecho en cuestión; c) propicia una mejor formación técnica y social: el investigador se ve obligado a ejercitar y adquirir continuamente conocimientos teórico-prácticos, en forma intensiva y, a la vez, la relación frecuente con los campesinos le da la oportunidad de conocer sus problemas y de apreciar sus conocimientos y cualidades humanas, y d) requiere de menos recursos de toda índole. Se elaboró una guía en relación con la biología y usos del maguey, se seleccionaron informantes para entrevistar, y finalmente se ordenó la información para de este modo recurrir a ella con facilidad en el momento de elaborar los informes requeridos.

\section{RESULTADOS Y DISCUSIÓN}

Identidad taxonómica. Las 60 colectas obtenidas en el área de estudio corresponden, siguiendo a Gentry (1982), a las siguientes especies y subespecies del subgénero Agave: Agave salmiana Otto ex Salm, ssp. crassispina (Trel.) Gentry "maguey verde"; $A$. scabra Salm. ssp. potosiensis Gentry; A. parrasana Berger "maguey chato"; A. americana L. var. oaxacensis Gentry; A. mapisaga Trel., var. mapisaga "mexicano", "penca larga", "jilotillo"; A. salmiana Otto ex Salm, var. salmiana "cuerno", "blanco" y "chino".

Otros nombres reportados para el área de estudio son: Agave crassispina Trel. (Breitung, 1968), que Gentry (1982) considera como una subespecie de $A$. salmiana Otto ex Salm.; A. asperrima Jacobi (Breitung, 1968), que Gentry (1982) trata como sinónimo de A. scabra Salm-Dyck ssp. scabra; A. atrovirens Karw. (Rzedowski, 1957; Breitung, 1968) en la que autores anteriores incluían $A$. salmiana Otto ex Salm-Dyck como sinónimo y que Gentry (1982) considera como especie distinta; A. gracilispina Engelm (Breitung, 1968), que Gentry (1982) clasifica como sinónimo de $A$. americana L. var. americana; A. americana L., ssp. protoamericana Gentry (Gentry, 1982); A. macroculmis Tódaro (Gentry, 1982); A. salmiana var. angustifolia Berger (Gentry, 1982); y A. gracilispina Eng. ex Trel., A. salmiana var. gracilispina Roll. Goss (Breitung, 1968), que Gentry (1982) considera sinónimo de $A$. americana L., var. americana.

Distribución geográfica y ecológica regionales. La distribución de las especies y subespecies se registra en la fig. 2.

El Agave salmiana ssp. crassispina es la subespecie silvestre más abundante y de mayor importancia económica en el área de estudio, encontrándose gran diversidad de formas, sin que por ello cambien su nombre común. Se distribuye de 1800 a $2400 \mathrm{msnm}$, en regiones con clima BS de Köppen (seco estepario) en sus dos variantes: BS。 (seco) 


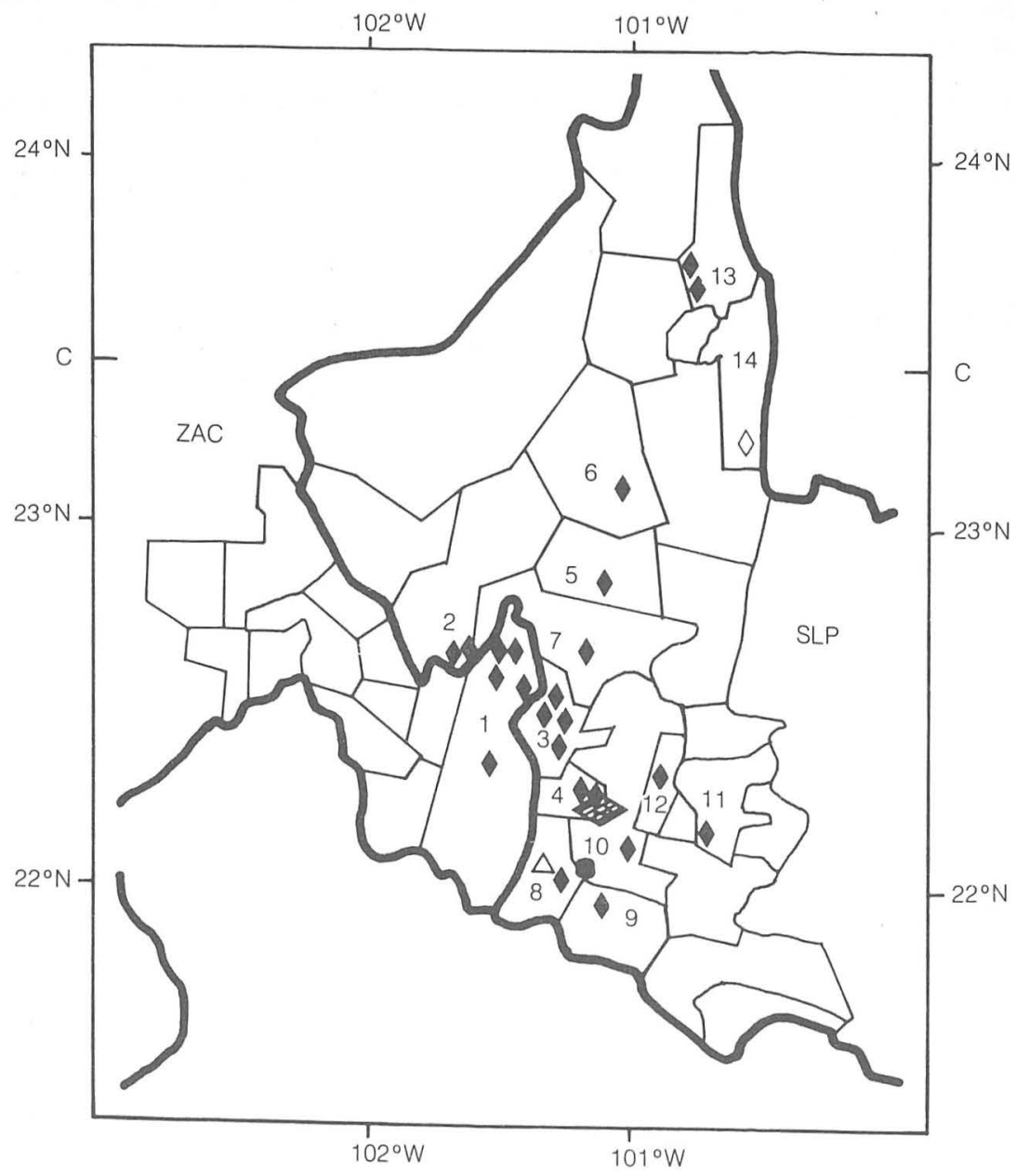

- Agave salmiana Otto ex Salm, ssp. crassispina (Trel.) Gentry

$\diamond$ Agave scabra Salm, ssp. potosiensis Gentry

$\triangle$ Agave americana L., var. oaxacensis Gentry

Agave mapisaga Trel., var. mapisaga

Agave salmiana Otto ex Salm, var. salmiana

- Agave parrasana Berger

1. Pinos; 2. Salinas de Hgo.; 3. Ahualulco; 4. Mexquitic; 5. Venado; 6. Charcas;

7. Moctezuma; 8. V. de Arriaga; 9. V. de Reyes; 10. San Luis Potosi; 11. Armadillo de los Infante; 12. Soledad D.G.; 13. Cedral; 14. Matehuala.

Fig. 2. Distribución de las especies y subespecies de maguey. 
y $\mathrm{BS}_{1}$ (semiseco) (García, 1973), con precipitaciones medias anuales de 325.8 a $502.9 \mathrm{~mm}$ y temperaturas medias anuales de 16 a $18.7^{\circ} \mathrm{C}$.

Las poblaciones se encuentran generalmente en pie de monte con ondulaciones moderadas y pendientes menores de $8 \%$ donde domina el material coluvio-aluvial (ígneo y sedimentario); predomina el suelo Litosol eútrico de textura media. Esta especie constituye un elemento del matorral crasicaule y del matorral micrófilo.

Donde la densidad de plantas es mayor existen mezcaleras. Este hecho se confirma con los datos de López et al. (1977) quien reporta 2275 plantas/ha en las inmediaciones de Santiago, Zacatecas. La densidad media de plantas por hectárea en cuatro localidades, es la siguiente: chicas, hasta $30 \mathrm{~cm}$ de altura, 1 098; medianas, de 30 a $60 \mathrm{~cm}$ de altura, 319 , y grandes, mayores de $60 \mathrm{~cm}$ de altura, 167; en total 1584 plantas/ha. Densidades de 1139 han sido reportadas por González (1982) en el Gran Tunal, Villa de Arriaga.

Agave scabra ssp. potosiensis, una subespecie silvestre de poca importancia económi$\mathrm{ca}$, se ubica en una pequeña zona del área de estudio localizada al SE de la cabecera municipal de Matehuala, SLP a 1330 msnm; con fórmula climática BS,kw"(e) (García, 1973), temperatura media anual de $19.3^{\circ} \mathrm{C}$ y precipitación de $442 \mathrm{~mm}$ anuales, en terreno plano con material aluvial y suelo xerosol gypsico de textura media. Esta especie es parte del matorral subinerme junto con izotales; en menor proporción se encuentran nopaleras aisladas.

Agave parrasana es una especie silvestre de poca importancia económica; se desarrolla en las partes altas de los bosques de pino-encino (2 $500 \mathrm{msnm})$ de las serranías meridionales al suroeste del estado de San Luis Potosí, entre los paralelos $21^{\circ} 51^{\prime}$ y $22^{\circ} 05^{\prime}$ latitud Norte y meridianos $100^{\circ} 55^{\prime}$ y $101^{\circ} 08^{\prime}$ longitud Oeste (Cetenal, 1973). El clima de la región es $\mathrm{BS}_{1} \mathrm{kw}(\mathrm{e}) \mathrm{g}$, templado semiseco, extremoso, oscilación de la temperatura entre 7 y $14^{\circ} \mathrm{C}$ con regímenes de lluvia de verano. La fisiografía del terreno es de lomeríos a terreno montuoso con pendientes entre 8 y 20 por ciento con material riolítico (ígneo) y suelo Litosol eútrico de textura media (Cetenal, 1973; García, 1973).

Agave americana var. oaxacensis, es una subespecie de poca importancia económica; semicultivada, ya que se aprecia que inicialmente se cultivó pero que a través del tiempo se ha propagado por sí sola, se desarrolla en una pequeña zona en las inmediaciones de El Tepetate, Villa de Arriaga, SLP, a $2120 \mathrm{msnm}$; con fórmula climática BS 1 kw"'i)g (García, 1973), temperatura media anual de $16.1^{\circ} \mathrm{C}$ y precipitación de $455 \mathrm{~mm}$ anuales; en sustrato ígneo (toba) y suelos de origen aluvial, Xerosol y Phaeozem háplicos de textura media; la vegetación nativa es un pastizal natural.

Agave mapisaga var. mapisaga y $A$. salmiana var. salmiana son subespecies que se cultivan en una pequeña zona al NNW de la ciudad de San Luis Potosí, entre los paralelos $22^{\circ} 11^{\prime}$ a $22^{\circ} 21^{\prime}$ latitud Norte y meridianos 100 $54^{\prime}$ a 101 ${ }^{\circ} 06^{\prime}$ longitud Oeste, a 1880 msnm (Cetenal, 1977), comprende parte de los municipios de Mexquitic de Carmona y San Luis Potosí, SLP. A. mapisaga var. mapisaga y dos formas de A. salmiana var. salmiana ("cuerno y blanco"), son encontradas en solares diseminados en el área de estudio. Las características climáticas corresponden a los BS de la clasificación de Köppen modificado por García (1973), la temperatura media anual es de $17.6^{\circ} \mathrm{C}$ y la precipitación de $402.7 \mathrm{~mm}$ anuales; con sustrato ígneo (toba) y suelo Xerosol háplico de textura media; terreno plano ligeramente ondulado con pendientes menores de $8 \%$. Estas subespecies se encuentran en terrenos abiertos al cultivo; la vegetación nativa es un matorral crasicaule muy deteriorado. 
Gentry (1982), señala que estas subespecies cultivadas fueron introducidas al altiplano después de la Conquista, cuando los españoles llegaron a colonizar regiones del norte, como Durango y Coahuila, llevando a los nahuatlacas, que utilizaban como intérpretes, trabajadores y agricultores, y quienes llevaron el maguey con ellos, estableciendo cultivos para producción de pulque.

Formas de utilización y conocimiento empírico. Mezcal. Es el nombre que se le da a una bebida alcohólica (aguardiente) obtenida por la destilación de los fermentos de los jugos provenientes de las "cabezas" cocidas (tallo y sección basal de las hojas) de ciertos agaves silvestres y cultivados. A las cabezas cocidas se les conoce como mezcal, del cual toma su nombre la bebida.

La principal zona mezcalera se encuentra al NW de San Luis Potosí, SLP, entre los paralelos $22^{\circ} 20^{\prime}$ a $22^{\circ} 38^{\prime}$ latitud Norte y meridianos $101^{\circ} 15^{\prime}$ a $101^{\circ} 42^{\prime}$ longitud Oeste (Cetenal, 1977). La mayor parte de esta zona corresponde a la jurisdicción del municipio de Pinos, Zac., y en menor proporción a los de Villa Hidalgo, Zac., y Ahualulco, SLP. Aquí se localizan las siguientes mezcaleras: La Pendencia, El Chino, Santiago y Saldaña del municipio de Pinos, Zac.; La Encarnación (Santa Teresa) del municipio de Ahualulco, SLP. Otra pequeña zona se localiza al NE de la cabecera municipal de Charcas, SLP, entre los paralelos $23^{\circ} 08^{\prime}$ a $23^{\circ} 15^{\prime}$ latitud Norte y meridianos $100^{\circ} 45^{\prime}$ a $101^{\circ} 00^{\prime}$ longitud Oeste (Cetenal, 1976). Se encuentra una sola mezcalera en operación en el rancho Santa Isabel (fig. 3).

El maguey utilizado como materia prima para la elaboración de mezcal es el Agave salmiana ssp. crassispina, conocido comúnmente como maguey verde. Esta planta crece en forma silvestre en estas zonas, pero para sostener la demanda es necesario su cultivo.

Para la obtención de materia prima de buena calidad, es esencial efectuar la castración o "picazón" del maguey, que consiste en la eliminación del brote o yema apical. A las personas encargadas se les denomina "picadores". Para determinar cuándo hay que efectuar la picazón se toman en consideración cambios en las características morfológicas de la planta, como es el adelgazamiento del "cogollo" (agrupamiento de hojas en forma cónica invertida, en el ápice del tallo; también se le denomina cogollo al brote que emerge del interior de estas hojas), el tamaño pequeño de las espinas del cogollo, el adelgazamiento y la dirección erecta de las hojas que envuelven a éste. La indicación más segura sobre este punto es la púa o espina del cogollo, la cual debe estar chica, negra y delgada. La planta que reúne estas características se denomina "maguey acarrillado". La edad de la planta al momento del acarrillado varía de ocho a 15 años, dependiendo de las condiciones del sitio. Según los datos de E. Palmer (Bye, 1979), la maduración ocurre en un periodo de ocho a 10 años.

El periodo que duran los magueyes castrados hasta el "rajado" (recolección) es muy variable, dependiendo de la programación del trabajo de las mezcaleras. El tiempo mínimo recomendado por los informantes es de 18 meses, tiempo durante el cual se da el sazonamiento o engrosamiento de la "piña" o cabeza del maguey. La práctica común es empezar a rajar de los seis a los 12 meses después de la castración.

A las personas encargadas de la recolección se les denomina "rajadores", quienes se integran generalmente en puntos de tres; un "desvirador" es el encargado de localizar las plantas castradas para, con una cuchilla, cortarle el mayor número posible de hojas o "pencas" que circundan la piña. A esta persona le siguen los "tumbadores", encarga- 

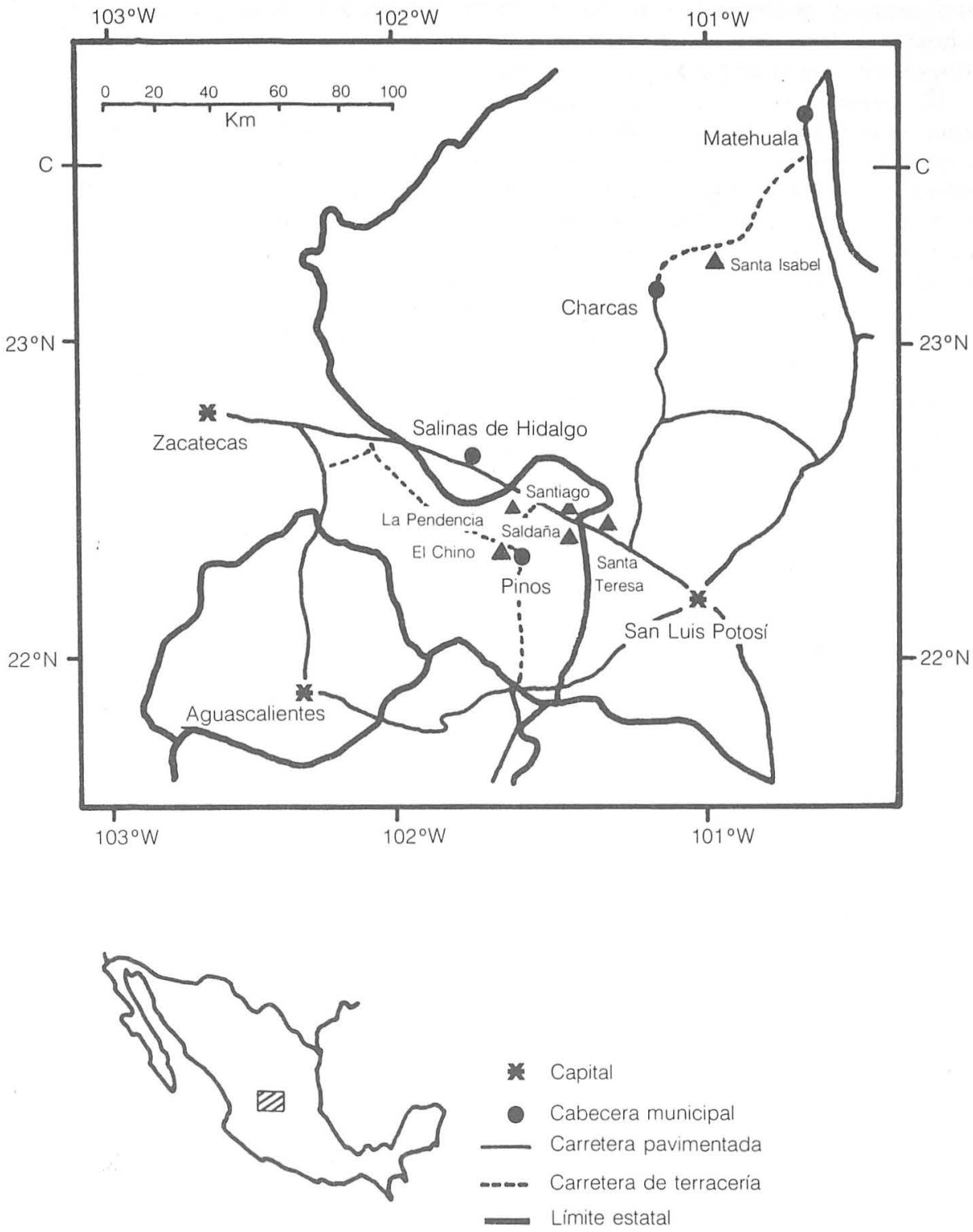

- Fábrica de mezcal

Fig. 3. Localización de las fábricas de mezcal. 
dos de arrancar la piña del suelo con una "barra tumbadora", lo que les permite cortar las hojas sobrantes con una cuchilla cortadora, y poner la piña en condiciones de ser transportada. Las mezcaleras pagan al ejido como máximo 14 pesos por piña.

Dentro de las observaciones realizadas en las diferentes mezcaleras, se aprecia el mal manejo común que se le da al recurso, ya que no es aprovechado de una manera planeada, en el sentido de programar la repoblación y las castraciones para obtener en una forma continua materia prima de buena calidad que haya pasado como mínimo 18 meses de "sazonamiento". Esto se debe quizá al poco interés de invertir a largo plazo, buscando las ganancias a corto plazo sin tomar en consideración, por un lado, el subaprovechamiento y, por otro, la sobreexplotación del recurso.

Otra evidencia del subaprovechamiento es la cantidad de hojas que son tumbadas de la piña y abandonadas en el campo. Esto es importante, ya que la cosecha mensual de plantas para mezcal se estima aproximadamente en 50000 . La baja proporción de la biomasa aérea utilizada y algunas características de los componentes de las plantas se muestran en el cuadro 1. La estimación del peso de las hojas desaprovechadas en el total de las mezcaleras es de 2270 toneladas mensuales.

CUADRO 1. Algunos valores de los componentes del maguey castrado

(12 meses), resultado de 50 observaciones realizadas en el ejido Caballerías, Villa Hidalgo, Zac.

\begin{tabular}{lcccc}
\hline Variable & Media & $\begin{array}{c}\text { Desviación } \\
\text { estándar }\end{array}$ & Mínimo & Máximo \\
\hline & $(\mathrm{kg})$ & $(\mathrm{kg})$ & $(\mathrm{kg})$ & $(\mathrm{kg})$ \\
\cline { 2 - 5 } & 111.2 & 51.4 & 36.0 & 247.5 \\
Peso de la planta & 65.8 & 31.9 & 21.0 & 166.0 \\
Peso de la piña & 45.4 & 23.4 & 8.0 & 110.0 \\
Peso de las hojas & 25.3 & 7.0 & 10.0 & 42.0 \\
Número de hojas & 2.2 & 1.9 & 0 & 8.0 \\
Número de hijos $<60 \mathrm{~cm} /$ planta & 0.8 & 1.2 & 0 & 5.0 \\
Número de hijos $>60 \mathrm{~cm} /$ planta & & & & \\
\hline
\end{tabular}

Una vez obtenidas las piñas, que contienen sustancias almidonosas de reserva, son sometidas a cocción prolongada en hornos durante 3 a 5 días, hidrolizándose los azúcares complejos que pasan al estado de manosas. Los hornos son de piedra con capacidades de 20 a 60 toneladas, con fuente de calor a base de fuego directo producido por combustóleo o vapor de agua generado por una caldera con fuente de calor a base de combustóleo o "mezotes" (hojas secas de maguey). A las piñas horneadas las denominan "mezcal"; éstas son despedazadas con hacha para facilitar su molienda en molinos de piedra con tracción animal o mecánica, y en trapiches con motores eléctricos; el mezcal exprimido es vuelto a remojar (lavado) y reexprimido en prensas o rodillos, hasta que se considera haber extraído la mayor cantidad de miel. El bagazo sobrante es retirado al patio de la mezcalera.

Las mieles obtenidas son recolectadas en pilas para su fermentación. Existen dos 
tipos de fermentación: natural y acelerada; en la primera, la miel es fermentada sin añadirle incitadores y el tiempo de fermentación es de tres días. En la segunda se agrega a la miel un pie de fermento o "xinaxtli" y un "puño" de sulfato de amonio. En este caso el tiempo de fermentación es de 12 a 24 horas.

La miel fermentada es destilada en alambiques de cobre. Los conderisados obtenidos generalmente son regulados a $45^{\circ} \mathrm{GL}$. El control de calidad en la regulación de las "cabezas" y las "colas" (primeros y últimos escurrimientos del alambique) son deficientes. El mezcal se almacena en recipientes de fibra de vidrio o en grandes tambos de madera denominados "pilones", de donde se vende a granel o se embotella. El precio de venta por litro a granel es de 1000 pesos y embotellado de 1500 pesos.

Procedimientos de destilación muy similares para la elaboración de mezcal en San Pedro y Ánima, Coahuila, han sido reportados por Bye (1979).

Ruvalcaba M. (1983) menciona que en Epazoyucan el maguey silvestre, conocido como "cimarrón", se somete a un proceso semejante al del agave mezcalero, obteniéndose un licor de alta graduación, de consumo local.

Mezcal campanilla. Anteriormente en el área de estudio se producía, clandestinamente y con tecnología muy imperfecta, una gran cantidad de licor conocido como "mezcal campanilla", elaborado con el líquido obtenido de las piñas cocidas del Agave salmiana ssp. crassispina y pulque $\operatorname{del} A$. mapisaga var. mapisaga y $A$. salmiana var. salmiana. Actualmente esta actividad está penada por la ley debido, principalmente, a la evasión de impuestos. A pesar de esto, un reducido número de campesinos, obligados por las circunstancias económicas, elaboran este tipo de licor. Esta actividad también ha sido mencionada por Bye (1979).

Bahre y Bradbury (1980) reportan un proceso similar en la elaboración del mezcal "bacanora", en Sonora, en el que se utiliza Agave pacifica y A. palmeri.

Pulque. Es una bebida espiritosa, producto de la fermentación de la savia conocida como "aguamiel" que se obtiene de algunas especies del género Agave.

Para la elaboración de pulque los campesinos obtienen el aguamiel de las plantas que han sido plantadas ex profeso. Las especies utilizadas en orden de importancia son: A. mapisaga var. mapisaga, A. salmiana var. salmiana en sus tres formas: maguey blanco, cuerno y chino, en orden inverso a lo reportado por Gentry (1982) para la Mesa Central.

Debido a que las lluvias se presentan principalmente en verano y algunas en noviembre y diciembre, la plantación se hace en los meses de marzo, abril, mayo, septiembre y octubre, haciéndose el mayor número de trasplantes en el mes de abril. Para las plantaciones se utilizan hijuelos de plantas adultas que no estén en explotación o en las que la floración se haya iniciado; se utilizan principalmente "hijos de primera", es decir, los primeros hijuelos que le brotan a la planta y que han alcanzado la altura de una vara $(.836 \mathrm{~m})$. Son obtenidos de plantas aledañas al lugar donde se realiza la plantación. Cuando en las plantaciones se utilizan hijos de magueyes en explotación (extracción de aguamiel) o plantas en proceso de formar su inflorescencia no desarrollan un tamaño normal, denominándolos "magueyes enanos" que únicamente se "aviejan". Para el desahije, a la planta madre se le cortan las hojas secas inferiores o "tacote" que estorbarían en esta actividad; ésta quizá sea la única poda que se le efectúa a la planta. Con una barra son desprendidos los hijuelos; con una cuchilla se le quitan las hojas secas inferiores y las 
raíces largas, procurando no dañar las yemas de nuevas raíces, y se efectúa el corte de las hojas hacia las raíces; después son oreados por cinco días con la finalidad de lograr la cicatrización de las heridas.

La plantación se efectúa en hileras enmarcando pequeñas áreas agrícolas de temporal; la planta es acomodada en la cepa tapando la parte inferior de la cabeza con tierra, presionándola con el pie; la separación de las cepas, las cuales se hacen al tanteo, es de aproximadamente $1.50 \mathrm{~m}$, y no efectúan ningún tipo de fertilización y abonado.

En las áreas agrícolas mencionadas, con menos potencial para la producción de cosechas, y aun en las mejores, se ha optado por remplazar los cultivos de maíz, frijol, trigo y cebada por el cultivo de maguey, que resulta más seguro sobre todo por su resistencia a la sequía.

Para la producción de aguamiel es necesario efectuar la castración o "rebullido", que consiste en la destrucción del inicio del tallo floral y la formación de una cavidad donde se colecte el aguamiel. Esta actividad se efectúa con más frecuencia antes de los periodos de lluvia, en los meses de marzo, abril y septiembre.

Una vez castrado, el maguey es dejado sazonar durante seis meses, con la finalidad de aumentar el tamaño del tallo y la calidad del aguamiel que produce, con respecto a su contenido de azúcares, así como para mantener la continuidad de la explotación. Es también común empezar a explotar el maguey después del tercer día de castrado, debido a la necesidad económica del campesino y a la deficiente programación de la explotación.

Para iniciar la explotación, la tasa o hendidura de la castración es desprovista de hojas y de costras formadas ("picazón"). La extracción del aguamiel de la hendidura de la planta se realiza con un "acocote" (Lagenaria vulgaris). Una vez extraído todo el aguamiel, se efectúa el "raspado" que consiste en retirar con un "raspador", una pequeña capa de tejido parenquimatoso de la hendidura, con la finalidad de evitar la cicatrización de los vasos que vierten sus líquidos en el depósito. El raspador es un implemento de fierro en forma de cuchara achatada con filo en sus bordes y con mango de madera. En una planta recién castrada el aguamiel fluye regularmente después de tres días de raspado; en los sazonados durante seis meses, es necesario rasparlos toda una semana. El periodo de producción es de tres a cuatro meses y son raspados tres veces al día. El frío afecta negativamente la producción, raspándose dos veces al día de noviembre a febrero. La producción es muy variable; una estimación es de tres litros diarios. Producciones de cinco a 12 litros de aguamiel son reportadas por Ruvalcaba (1983) para los magueyes de Epazoyucan, destacando el maguey "ayoteco" con ocho a 12 litros.

El aguamiel recolectado es colocado y vaciado en una olla de barro, la cual contiene pulque fuerte denominado xinaxtli o pie de fermento; ahí se va fermentando y transformando en pulque; en este recipiente se va vertiendo el producto de las tres colectas efectuadas en el día; cuando se saca el pulque para la venta, al día siguiente, se conserva una cantidad suficiente que sirve a su vez de xinaxtli.

El pulque es vertido en barricas de madera para transportarlo en motocicletas o bicicletas a los lugares de consumo. Generalmente se vende al menudeo por el propio productor en la periferia de San Luis Potosí. El precio de venta por litro es de 150 a 250 pesos "dependiendo del cliente"; cuando no se tiene vehículo de transporte, se entrega para su venta al vendedor que pueda transportarlo o a alguna pulquería, obteniendo ingresos de 50 pesos por litro. 
Cabe mencionar que en lustros anteriores la elaboración de pulque, en esta zona, era más tradicional; la recolección del aguamiel se hacía en "botas" de piel de cabra sin costura y la fermentación se efectuaba en recipientes hechos de cuero de bovino; además, utilizaban como ingredientes raíces de mezquite (Prosopis spp.) o garabatillo (Mimosa biuncifera), las que machucaban formando un manojo que era introducido en el aguamiel, obteniéndose un pulque baboso.

Miel de aguamiel. El aguamiel, obtenido en la misma forma ya descrita para la producción de pulque, se pone en cocimiento prolongado en un cazo de cobre colocado sobre una hornilla de adobe, atizado con leña u hojas secas de maguey (mezotes), para que se vaya evaporando. La miel es producida principalmente por campesinos que tienen en su solar plantas de Agave salmiana var. salmiana y A. mapisaga var. mapisaga. La elaboración de miel se hace únicamente en la Cuaresma. Es utilizada para las capirotadas y para la elaboración de dulces como el de biznaga. Esta forma de aprovechamiento también la reporta Bye (1979) para el área de estudio.

Quiote. El pedúnculo floral que le emerge a las plantas de $A$. salmiana ssp. crassispina que no fueron castradas es conocido como "quiote", el cual se aprovecha como golosina; para esto se cortan los pedúnculos florales que no han formado la "manilla" o panícula, la sección meristemática o punta se desecha; la porción obtenida, una vez que se pela (eliminación de brácteas), es transportada al cocedor, en donde se someten a cocimiento por un periodo de tres días. Este tipo de aprovechamiento ratifica lo consignado por Bye (1970). La época de quiote comprende los meses de octubre a mayo; ésta depende de la fenología de la floración. Es vendido en los poblados y las ciudades de la región. Un quiote vendido al menudeo en pequeños tajos proporciona ingresos de 2500 pesos.

Asimismo, en estos cocedores se acomodan las secciones basales de las hojas y el tallo de la planta para su cocción, obteniéndose el mezcal; éste, al igual que el obtenido de la cocción de las piñas para el licor de mezcal, se utiliza en forma secundaria para la alimentación humana y animal. A diferencia de lo reportado por Bye (1979) para esta misma región o para los tarahumaras y los seris (Bye et al., 1975; Felger y Moser, 1970), constituye un aprovechamiento sustantivo que produce alimento abundante y de buena calidad.

Forraje. El maguey silvestre Agave salmiana ssp. crassispina y los cultivados A. mapisaga var. mapisaga y $A$. salmiana var. salmiana, son las especies de mayor utilización forrajera en la cuenca lechera del valle de San Luis Potosí.

Las hojas o "pencas" de maguey silvestre son vendidas a pie de establo, por camiones introductores y carretoneros que regularmente hacen la entrega. Los camioneros las obtienen de los ejidatarios que cuentan con este recurso. Generalmente se aprovechan plantas grandes a las que les faltan algunos años para enquiotillar, lo que ocasiona una deforestación y un deterioro del medio donde se desarrollan al dejar desprotegidas a plantas de menor tamaño, además de que se ve afectada negativamente la producción de hijuelos.

Las pencas que son abandonadas en el campo, como consecuencia de la extracción de piñas para mezcal, no son aceptadas por los ganaderos, arguyendo que éstas no tienen la sección basal blanquecina o "migajón". Considerando la cantidad estimada de 2270 
toneladas mensuales de estas pencas, resultaría interesante investigar si este rechazo es consecuencia de su aspecto visual o debido a la mala calidad de éstas. Se realizó un análisis bromatológico, encontrándose en lo referente a proteína cruda (base seca), 4\% para la penca de desperdicio y $3.4 \%$ para la penca completa.

Las hojas de maguey cultivado son obtenidas, principalmente, en la zona pulquera localizada al NNW de la ciudad de San Luis Potosí, y en menor proporción de los campesinos que tienen en sus solares algunas de estas plantas. Existen dos tipos: las provenientes de magueyes explotados o raspados y las de plantas enquiotilladas. Las primeras son obtenidas cuando la planta ha dejado de producir aguamiel, y cuando el propietario, que por lo general es también ganadero, no las requiere para alimentar a sus animales. Las últimas son de magueyes enquiotillados, de plantas de buen tamaño que todavía no se les extrae el aguamiel, y que el campesino vende por necesidad.

La manera de preparar las pencas para su consumo es cortarle las espinas con una cuchilla y picarlas en pequeños pedazos que puedan ser ingeridos por el animal; la forma más típica de suministrar el forraje al ganado consiste en dar por la mañana maguey y un poco de salvado, posteriormente alfalfa y por la tarde maguey o nopal. El suministro se hace a todo tipo de animales (vacas en producción, secas, becerros, etc.). El ganadero obtiene el maguey por 5000 pesos la tonelada.

En la ganadería extensiva, el maguey (Agave salmiana ssp. crassispina) es picado en el campo por los pastores; en época de seca el maguey y el nopal son los únicos forrajes disponibles que hacen posible la subsistencia de esta ganadería.

Gentry (1982) reporta que en San Luis Potosí las pencas de maguey son aprovechadas como forraje, estimando su utilización en varios miles de toneladas por año; señala asimismo que es un aprovechamiento que requiere investigación.

Martínez V. (1983) considera que $91 \%$ de los estableros de la cuenca lechera del valle de San Luis Potosí utilizan maguey como ingrediente principal de las raciones alimenticias. El mismo autor (comunicación personal) estima un aprovechamiento del orden de $150 \mathrm{ton} / \mathrm{semana}$ de pencas de maguey.

Las explotaciones extensivas de ganado bovino aledañas a las mezcaleras utilizan como forraje el bagazo desperdiciado en la elaboración de mezcal, principalmente en la época de seca. Las explotaciones de engorda de ganado bovino estabulado en el municipio de Salinas de Hidalgo, SLP, utilizan una ración empírica variable, a base de bagazo de maguey, sorgo y gallinaza. El precio por tonelada de bagazo en la mezcalera es de 2000 pesos.

Se han realizado estudios con el bagazo de Agave spp. con fines forrajeros. Montes R. (1981) y Herrera D. (1984) trabajaron con materiales de Agave tequilana y A. salmiana ssp. crassispina, respectivamente. Los autores reportan que pueden sustituir hasta en $60 \%$ los forrajes toscos como el rastrojo de maíz utilizado en raciones para rumiantes, y que, con un suplemento proteínico-energético, resulta un buen alimento que no causa efectos negativos en el comportamiento animal.

Construcción. Todo tipo de quiotes son utilizados para diferentes construcciones. Al maguey "chino" (Agave salmiana var. salmiana) se le deja deliberadamente que le emerja el quiote, ya que éste es muy apreciado por su solidez y resistencia. Las hojas son utilizadas en la construcción de techos de jacales. 
Ornamental. Los tallos de los magueyes pulqueros después de haber sido raspados y eliminados de sus hojas son utilizados como macetas. La inflorescencia de los diferentes magueyes en fruta o ya seca es utilizada como árbol navideño.

Usos varios. Sin especificar especies, se refieren usos tales como: elaboración de pan, medicina, andamios, escaleras, apiarios, nidos de gallina, bancos, aguamiel hervida y enfriada en cántaros de barro, cercas vivas, etcétera.

\section{CONCLUSIONES}

1. Las especies y subespecies de Agave (subgénero Agave) silvestres registradas para el área son: Agave salmiana ssp. crassispina, $A$. scabra ssp. potosiensis y $A$. parrasana; semicultivadas: $A$. americana var. oaxacensis; cultivadas: $A$. mapisaga var. mapisaga y $A$. salmiana var. salmiana.

2. A. salmiana ssp. crassispina (maguey verde), es la de mayor distribución e importancia económica, siendo utilizada en la elaboración de mezcal y en la preparación de quiote; también como forraje, en construcción, como planta ornamental, como material combustible, en conservación de suelos y para cercas vivas.

$A$. mapisaga var. mapisaga y $A$. salmiana var. salmiana son utilizadas en la extracción de aguamiel para la elaboración de pulque y miel, como forraje, en construcción, como planta ornamental y en cercas vivas.

3. Existe un subaprovechamiento en las formas de utilización del maguey, ya que los beneficios que de él se obtienen podrían incrementarse al mejorar las prácticas de manejo y los procesos de transformación.

4. Las poblaciones de $A$. salmiana ssp. crassispina están disminuyendo a consecuencia del desequilibrio entre uso y replantación.

5. Las formas de utilización del maguey están sustentadas en el conocimiento empírico; proveen de ingresos a la población rural, pero en algunos casos están en peligro de desaparecer. Un uso planificado úe este recurso, basado en la biología de la planta en combinación con el conocimiento empírico existente, puede resultar en la integración de una utilización por largo tiempo.

\section{LITERATURA CITADA}

Bailey, L.H., 1949. Manual of cultivated planis. 2a. ed. MacMillan Publishing Co., Inc. New York.

Bahre, D.J. y D.E., Bradbury, 1980. Manufacture of mescal in Sonora, Mexico. Econ. Bot. 34(4):391-400. Bazant, J., 1975. Cinco haciendas mexicanas. El Colegio de México. México. 226 pp.

Breitung, A.J., 1968. The Agaves. Cact. Succ. J. Yearbook, 1968:1-107. (Reimpreso de Cact. Succ. J., vols. 31-38). Bye, R., D. Burgfss y A.T. MAREs, 1975. Ethnobotany of the western Tarahumara of Chihuahua. 1. Notes on the genus Agave, Bot. Mus. Leafl. 24:85-112.

1979. An 1878 Ethnobotanical collection from San Luis Potosi: Dr. Edward Palmer's First Major Mexican Collection. Econ. Bot. 33(2):135-162.

Cetenal, 1970. Carta de Climas, 140-1, escala 1:500 000. Comisión de Estudios del Territorio Nacional. Secretaría de la Presidencia. México.

1973. Cartas: topográfica, geológica, edafológica y uso del suelo, F-14-A-83, escala 1:50 000. Comisión de Estudios del Territorio Nacional. Secretaría de la Presidencia. México. 
1976. Cartas topográficas F-14-1, escala 1:250 000. Comisión de Estudios del Territorio Nacional. Secretaría de la Presidencia. México.

1977. Cartas topográficas F-14-4, F-14-7, escala 1:250 000. Comisión de Estudios del Territorio Nacional. Secretaría de la Presidencia. México.

CuE, C.A., 1979. Historia social y económica de México. 1521-1584. Ed. Trillas, México. 422 pp.

Felger, R. y M.B. Moser, 1970. Seri use of Agave (century plant). Kiva 35:159-167.

GARCíA, E., 1973. Modificaciones al sistema de clasificación climática de Köppen. Instituto de Geografía, UNAM, México. 246 pp.

García, M.E. y J. Villa V., 1977. Factores ambientales que afectan la distribución geográfica y ecológica de Boutelona gracilis (H.B.K.) Lag. ex Steud, en el estado de San Luis Potosí. Agrociencia 28:3-29.

Gentry, H.S., 1976. La simbiosis hombre-Agave. Cact. Suc. Mex. 21(1):16-23. , 1982. Agaves of Continental North America. The University of Arizona Press. Tucson, Arizona. 670 pp.

GONZÁLEZ, E.M., 1982. Seed predation by desert harvester ants and rodents in central Mexico. Dissertation. University of Pensylvania, Filadelfia, Pennsilvania. $180 \mathrm{pp}$.

Guerrero, G.R., 1980. El pulque. Instituto Nacional de Antropología e Historia. México. 241 pp.

HERnÁnDEZ, X.E., y A. RAmos R., 1977. Metodología para el estudio de agroecosistemas con persistencia de tecnología tradicional. En: E. Hernández X. (ed.), Agroecosistemas de México. Colegio de Postgraduados, Chapingo, México, pp. 321-333.

Herrera, D.D., 1984. Efecto de la suplementación con gallinaza sobre el valor nutritivo del bagazo del maguey (Agave salmiana) para ovinos. Tesis de maestría, Colegio de Postgraduados, Chapingo, México. 94 pp.

Lezama, M.M., 1975. El maguey. El Campo 3-20.

López, C.J.J., J.M. Gasto C., R. Nava C. y J.C. Medina T., 1977. Ecosistema Opuntia steptacantha Lemaire. UAAAN. Monografía técnico-cientifica 3(5):394-545.

Martínez V.E., 1983. Análisis de los sistemas de producción en la cuenca lechera del Valle de San Luis Potosí. Tesis, Escuela de Agronomía, UASLP, San Luis Potosí, México.

Miranda, F. y E. HernÁNDEZ X., 1964. Fisiografía y vegetación. En: E. Beltrán (ed.), Las zonas áridas del centro y noreste de México y el aprovechamiento de sus recursos. IMRNR. México. 1-28 pp.

MONTES R.A., 1981. Evaluación del bagazo de maguey tequilero (Agave tequilana) como ingrediente en raciones para rumiantes. Tesis de maestría, Colegio de Postgraduados, Chapingo, México, 80 pp.

Ruvalcaba M.J., 1983. El maguey manso. Colección de Cuadernos Universitarios. Serie: Ciencias Sociales núm. 4. Universidad Autónoma de Chapingo, México. 122 pp.

RzEDOwsKi, J., 1955. Notas sobre la flora y la vegetación del estado de San Luis Potosí, II. Estudio de diferencias florísticas y ecológicas condicionadas por ciertos tipos de sustrato geológico. Ciencia (México). 15(6-8):141-158.

1957. Vegetación de las partes áridas de los estados de San Luis Potosí y Zacatecas. Rev. Soc. Mex. Hist. Nat. 18(1-4):49-101.

1965. Vegetación del estado de San Luis Potosí. Acta Cient. Potos. 5(1-2):5-291. , 1968. Las principales zonas áridas de México y su vegetación. Bios 1(1):4-24.

Sánchez-Marroquín, A., 1979. Los agaves de México en la industria alimenticia. Centro de Estudios Económicos y Sociales del Tercer Mundo. México. 526 pp. 